\title{
Convergence of chiral perturbation theory in dynamical lattice QCD with exact chiral symmetry
}

\author{
Jun-Ichi Noaki for the JLQCD and TWQCD Collaborations \\ High Energy Accelererator Research Organization (KEK), Tsukuba 305-0801, Japan \\ E-mail: hoakidpost.kek.ip
}

\begin{abstract}
We present our recent lattice calculation with dynamical quarks using the overlap fermion formulation, which has exact chiral symmetry. It is possible to compare our data of meson mass and decay constant with the prediction from the chiral perturbation theory. From such comparison, we investigate the convergence property of the chiral expansion. For $N_{f}=2$, we observe that the prediction to NLO does not converge at the scale of kaon mass. Based on this fact, we extend the analysis to the $N_{f}=2+1$ case and carry out the extrapolation to the physical mass point using the NNLO formulae.
\end{abstract}




\section{Introduction}

In lattice QCD, numerical simulations are carried out with quark masses given as simulation parameters. Since the quark mass around the physical value makes the cost of numerical simulation highly demanding, data are usually obtained at masses significantly heavier than those in nature. Results at the physical mass point are obtained by an extrapolation of the data points. It is therefore crucial for the accuracy of lattice calculation to make a reliable extrapolation in the function of quark masses or, equivalently, in the pseudo-scalar quark masses. The chiral perturbation theory (ChPT) gives a theoretical guide for this extrapolation [四].

ChPT is an effective theory constructed from QCD based on the chiral symmetry and its spontaneous breaking. This theory describes the physics in the low-energy region $p^{2} \approx m_{\pi}^{2}$ where Nambu-Goldstone pions dominate the dynamics of the system. One of the characteristics of ChPT is that the Lagrangian is written in terms of an expansion in $p^{2}: \mathscr{L}=\mathscr{L}_{2}+\mathscr{L}_{4}+\cdots$, where $\mathscr{L}_{2 n}$ contains interactions among mesons of momenta $\mathscr{O}\left(p^{2 n}\right)$. Based on ChPT, physical quantities are thus expanded in $p^{2}$ and $m_{\pi}^{2}$. For the quantities such as meson masses and decay constants, the leading order (LO) contribution from $\mathscr{L}_{2}$ is corrected by the next-to-leading order (NLO) terms, which consist of one-loop effects with $\mathscr{L}_{2}$ and tree-level insertions of $\mathscr{L}_{4}$. It is possible to add even higher order corrections from multi-loop level diagrams and higher order Lagrangeans.

Often, lattice data are fitted using the NLO ChPT formula as it is the best known functional form for the quantity of interest. But, in many cases, chiral extrapolations are carried out in the mass region below the cut off $\Lambda_{\chi}=2 \sqrt{2} \pi f_{\pi} \approx 1.2 \mathrm{GeV}$ without checking the convergence of chiral expansion at NLO. For the kaon sector, in particular, the convergence at the kaon mass $m_{K} \approx 500$ $\mathrm{MeV}$ is a non-trivial issue. One of the problems for the convergence test is that conventional fermion formalisms on the lattice explicitly break chiral symmetry or flavor symmetry then allow for inconsistency between numerical data and continuum theory.

In this article, we present our study of the convergence property of ChPT using the numerical simulation with dynamical overlap fermion [[]], with which chiral symmetry as well as flavor symmetry is exactly preserved on the lattice. We generated two sets of gauge configurations with different number of flavors $N_{f}=2$ [园] and $2+1$ [四], on which a series of phenomenological quantities including the kaon $B$-parameter, $B_{K}$ [ [ $]$, have been calculated. We test the convergence of ChPT through the fit of light meson masses and decay constants, i.e. $m_{\pi}^{2}$ and $f_{\pi}$ for $N_{f}=2$ and $m_{\pi}^{2}$, $m_{K}^{2}, f_{\pi}$ and $f_{K}$ for $N_{f}=2+1$, and determine of the coupling constants of the ChPT Lagrangean, i.e. low-energy constants (LECs). We also obtain the fundamental physical quantities such as the chiral condensate, up and down quark masses and strange quark mass as a result of the chiral extrapolation.

In the following section, we briefly explain how to obtain the data points on the lattices, which is common for the calculations with different number of flavors. To discuss the issue of convergence, we describe a test of ChPT performed for the $N_{f}=2$ case [ [6] in Section [3. Based on this test, in Section 团, we present the chiral extrapolation of the $N_{f}=2+1$ data by using the NNLO ChPT formulae. 


\section{Getting data points}

We refer [B], 团] for the details of the generation of the gauge configurations. For $N_{f}=2$, we generate $10,000 \mathrm{HMC}$ trajectories on a $16^{3} \times 32$ lattice at six different sea quark masses $m_{\text {sea }}$ while, for $N_{f}=2+1$, we generate 2,500 trajectories on a $16^{3} \times 48$ lattice for ten combinations of up-down and strange sea quark masses, i.e. five $m_{u d}$ 's times two $m_{s}$ 's.

For $N_{f}=2(2+1)$, we calculate 50 (80) pairs of the lowest-lying eigenmodes on each gauge configuration and store them on the disks. These eigenmodes are used to construct the low-mode contribution to the quark propagators. The higher-mode contribution is obtained by conventional CG calculation with significantly smaller amount of machine time than the full CG calculation. Those eigenmodes are also used to replace the lower-mode contribution in the meson correlation functions by that averaged over the source location (low-mode averaging) [ $[\mathbb{Z},[]]$. We extract meson mass from the exponential decay of the time-separated correlation function of pseudo-scalar operator $\langle P(t) P(0)\rangle$. The decay constant, which is defined by the matrix element of the axial-current operator $\mathscr{A}_{\mu}$, is obtained simultaneously using the PCAC relation $\partial_{\mu} \mathscr{A}_{\mu}=2 m_{q} P$.

Throughout the Monte Carlo updates for both $N_{f}$ 's, the global topological charge of the gauge configurations is fixed to zero. This is necessary to avoid discontinuous change of the Dirac eigenvalue, which is numerically too-expensive. The artifact due to fixing the topology is understood as a finite size effect [ $[0]$ in addition to the conventional finite size effect. For the physical size of our lattice $L \approx 1.7 \mathrm{fm}$, the finite size effect could be sizable. We calculate both kinds of finite size effect from the analytic formulae based on ChPT [س], ㅁ] ]. In particular, for the effect of the fixed topology, we make use of the numerical data of the topological susceptibility determined on the same lattice configurations [12].

In order to obtain the physical quark mass, we need to renormalize bare quark mass on the lattice as $m_{q}^{(\text {ren })}=Z_{m} m_{q}^{(\text {bare })}$. We obtain the renormalization factor $Z_{m}$ by calculating scalar and pseudo-scalar vertex functions in the momentum space in the Landau gauge and applying the $\mathrm{RI} / \mathrm{MOM}$ scheme [133]. In extracting $Z_{m}$ from the vertex functions, we control the contamination from the spontaneous chiral symmetry breaking by using the the low-mode contribution to the chiral condensate [14]. Using the perturbative matching factor known to 4-loop level and the extrapolation to the chiral limit, i.e. $m_{\text {sea }}=0$ for $N_{f}=2$ and $m_{u d}=m_{s}=0$ for $N_{f}=2+1$, we obtain the final results of $Z_{m}$ in the $\overline{\mathrm{MS}}$ scheme at $2 \mathrm{GeV}$.

In the rest of this article, it is understood that all data points are corrected by the finite size effects and quark masses are renormalized.

\section{Convergence of ChPT $\left(N_{f}=2\right)$}

Determining the lattice scale by the Sommer scale with an input $r_{0}=0.49 \mathrm{fm}$, we obtain $a^{-1}=$ 1.667(17) GeV. It imply that our data points cover the pion mass region $290 \mathrm{MeV} \lesssim m_{\pi} \lesssim 750 \mathrm{MeV}$.

In the framework of $S U(2) \mathrm{ChPT}$ which describes the data with $N_{f}=2$, pion mass and decay constants are expanded in terms of $x=4 B m_{q} /(4 \pi f)^{2}$ as

$$
\begin{aligned}
m_{\pi}^{2} / m_{q} & =2 B\left(1+\frac{1}{2} x \ln x\right)+c_{3} x, \\
f_{\pi} & =f(1-x \ln x)+c_{4} x
\end{aligned}
$$



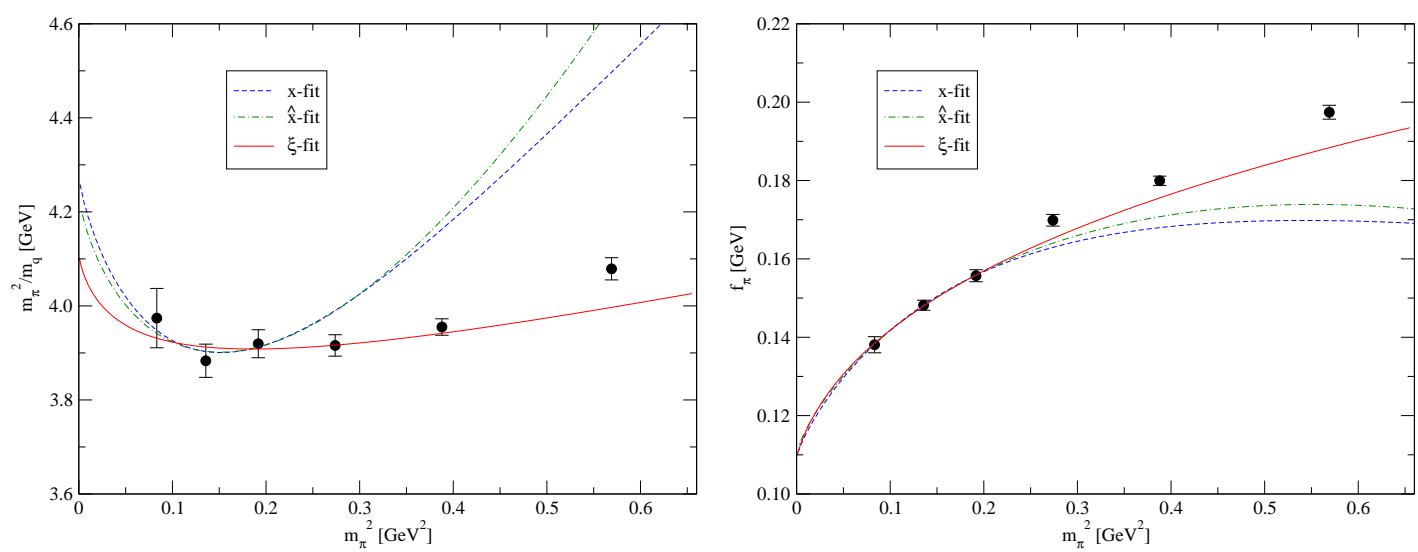

Figure 1: Chiral extrapolation of $m_{\pi}^{2} / m_{q}$ (left) and $f_{\pi}$ (right) using NLO ChPT formulae. The lightest three data points are used for the fit.

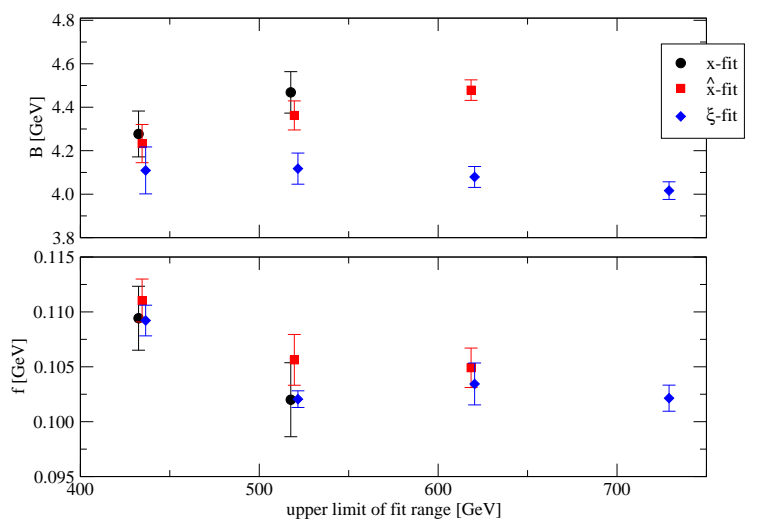

Figure 2: Results of fit parameters $B$ (top) and $f$ (bottom) as functions of the upper limit of the fit range. In each panel, circle, square and diamonds are obtained with fit parameters $x, \hat{x}$ and $\xi$. Results with $\chi^{2} /$ dof $\lesssim 2$ are plotted.

to NLO (i.e. one-loop level or $\mathscr{O}(x)$ ), where $B$ and $f$ are the tree level LECs, and $c_{3}$ and $c_{4}$ are related to the one-loop level LECs $\bar{l}_{3}$ and $\bar{l}_{4}$. At NLO, these expressions are unchanged when one replaces the expansion parameter $x$ by $\hat{x}=2 m_{\pi}^{2} /(4 \pi f)^{2}$ or $\xi=2 m_{\pi}^{2} /\left(4 \pi f_{\pi}\right)^{2}$, where $m_{\pi}^{2}$ and $f_{\pi}$ denote those at a finite quark mass. In other words, in a small enough pion mass region the three expansion parameters should describe the lattice data equally well.

Three fit curves corresponding to $x$-fit, $\hat{x}$-fit and $\xi$-fit for the three lightest pion mass points $\left(m_{\pi} \lesssim 450 \mathrm{MeV}\right)$ are shown in Figure $\mathbb{W}$ as a function of $m_{\pi}^{2}$. For all fits, the horizontal axis is appropriately rescaled to give $m_{\pi}^{2}$ using the obtained fit curves. From the plot we observe that the different expansion parameters describe the three lightest points equally well; the values of $\chi^{2} /$ dof are $0.30,0.33$ and 0.66 for $x$-, $\hat{x}$ - and $\xi$-fits, respectively. In each fit, the correlation between $m_{\pi}^{2} / m_{q}$ and $f_{\pi}$ for common sea quark mass is taken into account. Between the $x$ - and $\hat{x}$-fit, all of the resulting fit parameters are consistent. Among them, $B$ and $f$ are also consistent with the $\xi$-fit. This indicates that the NLO formulae successfully describes the data. In Figure $\square$, results of 


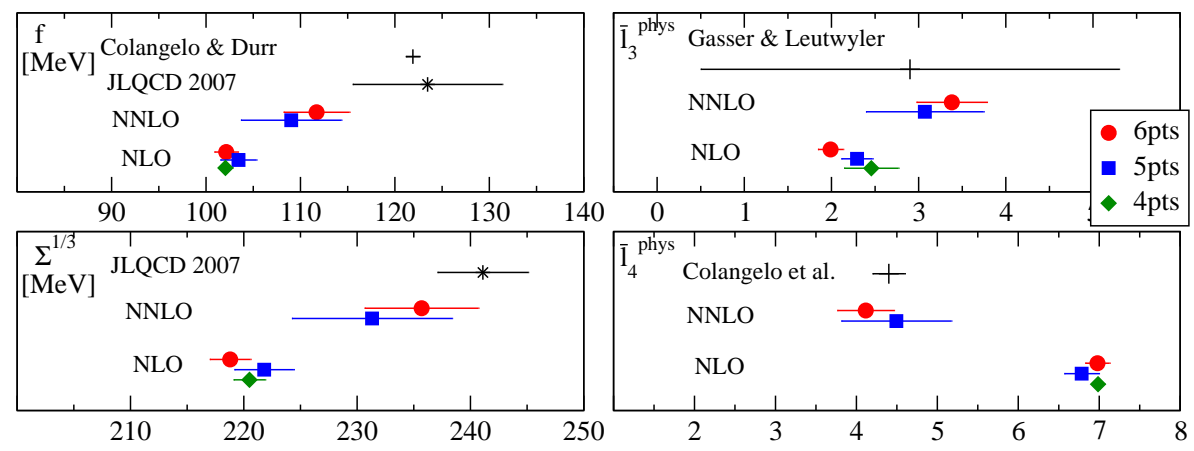

Figure 3: Comparison of the $N_{f}=2$ results from the NLO fit and the NNLO fit with $\xi$. Black pluses denote reference points from phenomenological estimations.

$B$ (upper panel) and $f$ (bottom panel) from the different fits are plotted for various fitting range. As seen in the figure, the agreement among the different expansion prescriptions is lost when we extend the fit range to include the 4 th lightest data point which corresponds to $m_{\pi} \simeq 520 \mathrm{MeV}$. We, therefore, conclude that for these quantities the NLO ChPT may be safely applied only below $\approx$ $450 \mathrm{MeV}$.

Another important observation from Figure $\mathbb{W}$ is that only the $\xi$-fit reasonably describes the data beyond the fitted region. With the $x$ - and $\hat{x}$-fits the curvature due to the chiral logarithm is too strong to accommodate the heavier data points. In fact, values of the LECs with the $x$ - and $\hat{x}$-fits are more sensitive to the fit range than the $\xi$-fit. This is because $f$, which is significantly smaller than $f_{\pi}$ of our data, enters in the definition of the expansion parameter. Qualitatively, by replacing $m_{q}$ and $f$ by $m_{\pi}^{2}$ and $f_{\pi}$, higher loop effects in ChPT are effectively resummed and the convergence of the chiral expansion is improved.

We then extend the analysis to include the NNLO terms [[5]:

$$
\begin{aligned}
m_{\pi}^{2} / m_{q} & =2 B\left[1+\frac{1}{2} \xi \ln \xi+\frac{7}{8}(\xi \ln \xi)^{2}+\left(\frac{c_{4}}{f}-\frac{1}{3}\left(\tilde{l}^{\text {phys }}+16\right)\right) \xi^{2} \ln \xi\right]+c_{3} \xi\left(1-\frac{9}{2} \xi \ln \xi\right)+\alpha \xi^{2}, \\
f_{\pi} & =f\left[1-\xi \ln \xi+\frac{5}{4}(\xi \ln \xi)^{2}+\frac{1}{6}\left(\tilde{l}^{\text {phys }}+\frac{53}{2}\right) \xi^{2} \ln \xi\right]+c_{4} \xi(1-5 \xi \ln \xi)+\beta \xi^{2}
\end{aligned}
$$

Since we found that only the $\xi$-fit reasonably describes the data beyond $m_{\pi} \simeq 450 \mathrm{MeV}$, we perform the NNLO analysis using the $\xi$-expansion. Although we input phenomenological estimate for the LEC $\tilde{l}^{\text {phys }}$, we find our fit result is insensitive to their uncertainties. We extract the LECs of ChPT, i.e. the decay constant in the chiral limit $f$, chiral condensate $\Sigma=B f^{2} / 2$, and the NLO LECs $l_{3}^{\text {phys }}=-c_{3} / B+\ln \left(2 \sqrt{2} \pi f / m_{\pi^{+}}\right)^{2}$ and $l_{4}^{\text {phys }}=c_{4} / f+\ln \left(2 \sqrt{2} \pi f / m_{\pi^{+}}\right)^{2}$. For each quantity, a comparison of the results between the NLO and the NNLO fits is shown in Figure [3]. In each panel, the results with 5 and 6 lightest data points are plotted for the NNLO fit. The correlated fits give $\chi^{2} /$ dof $=1.94$ and 1.40 , respectively. For the NLO fits, we plot results obtained with 4, 5 and 6 points to show the stability of the fit. The $\chi^{2} /$ dof is less than 1.94 . The results for these physical quantities are consistent within either the NLO or the NNLO fits. On the other hand, as seen for $\vec{l}_{4}^{\text {phys }}$ most prominently, there is a significant disagreement between NLO and NNLO. This is due to the large NNLO contributions to the terms which are proportional to $c_{3}$ and $c_{4}$, respectively. 

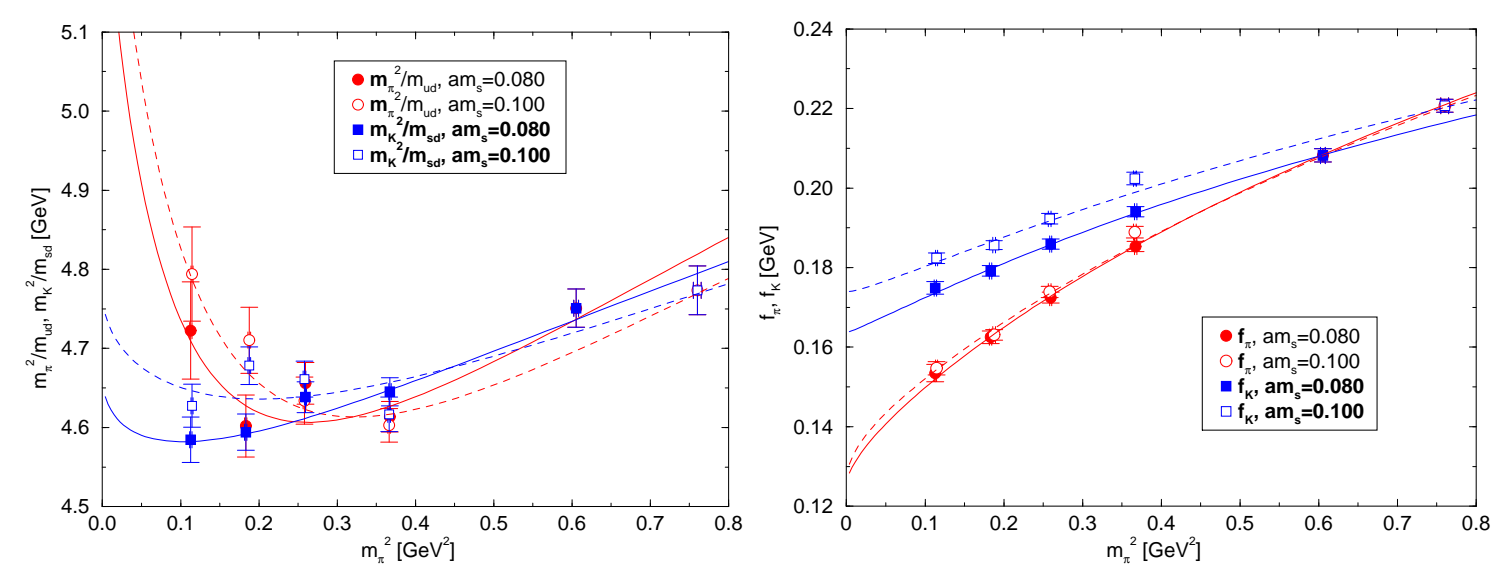

Figure 4: Chiral extrapolation using the NNLO full $S U$ (3) ChPT formulae for $m_{\pi}^{2} / m_{q}$ (circles) and $m_{K}^{2} / m_{s d}$ (squares) in the left panel and $f_{\pi}$ (circles) and $f_{K}$ (squares) in the right.

We quote our results for the $N_{f}=2$ calculation from the NNLO fit with all data points: $f=$ 111.7(3.5) $(1.0)\left({ }_{-0.0}^{+6.0}\right) \mathrm{MeV}, \Sigma^{\overline{\mathrm{MS}}}(2 \mathrm{GeV})=\left[235.7(5.0)(2.0)\left({ }_{-0.0}^{+12.7}\right) \mathrm{MeV}\right]^{3}, l_{3}^{\text {phys }}=3.38(40)(24)\left({ }_{-0}^{+31}\right)$, and $\bar{l}_{4}^{\text {phys }}=4.12(35)(30)\left(\begin{array}{c}+31 \\ -0\end{array}\right)$, where $m_{\pi}^{+}=139.6 \mathrm{MeV}$. From the value at the neutral pion mass $m_{\pi^{0}}=135.0 \mathrm{MeV}$, we obtain the average up and down quark mass $m_{u d}$ and the pion decay constant as $m_{u d}^{\overline{\mathrm{MS}}}(2 \mathrm{GeV})=4.452(81)(38)\left({ }_{-227}^{+0}\right) \mathrm{MeV}$ and $f_{\pi}=119.6(3.0)(1.0)\left({ }_{-0.0}^{+6.4}\right) \mathrm{MeV}$. In these results, the first error is statistical, where the error of the renormalization constant is included in quadrature for $\Sigma^{1 / 3}$ and $m_{u d}$. The second error is systematic due to the truncation of the higher order corrections. For quantities carrying mass dimensions, the third error is from the ambiguity in the determination of $r_{0}$. We estimate these errors from the difference of the results with our input $r_{0}=0.49 \mathrm{fm}$ and that with $0.465 \mathrm{fm}$. The third errors for $l_{3}^{\text {phys }}$ and $l_{4}^{\text {phys }}$ reflect an ambiguity of choosing the renormalization scale of ChPT $\left(4 \pi f\right.$ or $\left.4 \pi f_{\pi}\right)$.

\section{Results in the $N_{f}=2+1$ simulation}

\subsection{Fit to NNLO $S U(3)$ ChPT}

Since we found in the two-flavor calculation that the NNLO ChPT formulae can nicely fit our data even in the kaon mass region if one uses the $\xi$-expansion, we apply the same strategy for our $2+1$-flavor analysis. As functions of $\xi_{\pi}=2 m_{\pi}^{2} /\left(4 \pi f_{\pi}\right)^{2}$ and $\xi_{K}=2 m_{K}^{2} /\left(4 \pi f_{\pi}\right)^{2}$, predictions from the $S U(3)$ ChPT are expressed as

$$
\begin{aligned}
m_{\pi}^{2} / m_{u d} & =2 B_{0}\left[1+M^{\pi}\left(\xi_{\pi}, \xi_{K} ; L_{4}^{r}, L_{5}^{r}, L_{6}^{r}, L_{8}^{r}\right)\right]+\alpha_{1}^{\pi} \cdot \xi_{\pi}^{2}+\alpha_{2}^{\pi} \cdot \xi_{\pi} \xi_{K}+\alpha_{3}^{\pi} \cdot \xi_{K}^{2} \\
m_{K}^{2} / m_{s d} & =2 B_{0}\left[1+M^{K}\left(\xi_{\pi}, \xi_{K} ; L_{4}^{r}, L_{5}^{r}, L_{6}^{r}, L_{8}^{r}\right)\right]+\alpha_{1}^{K} \cdot \xi_{\pi}\left(\xi_{\pi}-\xi_{K}\right)+\alpha_{2}^{K} \cdot \xi_{K}\left(\xi_{K}-\xi_{\pi}\right), \\
f_{\pi} & =f_{0}\left[1+F^{\pi}\left(\xi_{\pi}, \xi_{K} ; L_{4}^{r}, L_{5}^{r}\right)\right]+\beta_{1}^{\pi} \cdot \xi_{\pi}^{2}+\beta_{2}^{\pi} \cdot \xi_{\pi} \xi_{K}+\beta_{3}^{\pi} \cdot \xi_{K}^{2} \\
f_{K} & =f_{0}\left[1+F^{K}\left(\xi_{\pi}, \xi_{K} ; L_{4}^{r}, L_{5}^{r}\right)\right]+\beta_{1}^{K} \cdot \xi_{\pi}\left(\xi_{\pi}-\xi_{K}\right)+\beta_{2}^{K} \cdot \xi_{K}\left(\xi_{K}-\xi_{\pi}\right)
\end{aligned}
$$

where $m_{s d}=\frac{1}{2}\left(m_{s}+m_{u d}\right)$ and $\alpha_{i}^{\pi, K}$ and $\beta_{i}^{\pi, K}$ are NNLO unknown parameters. Functions $M^{\pi}, M^{K}$, $F^{\pi}$ and $F^{K}$ contain the NLO contributions from $\mathscr{L}_{4}$ and the NLO and NNLO loop contributions, 

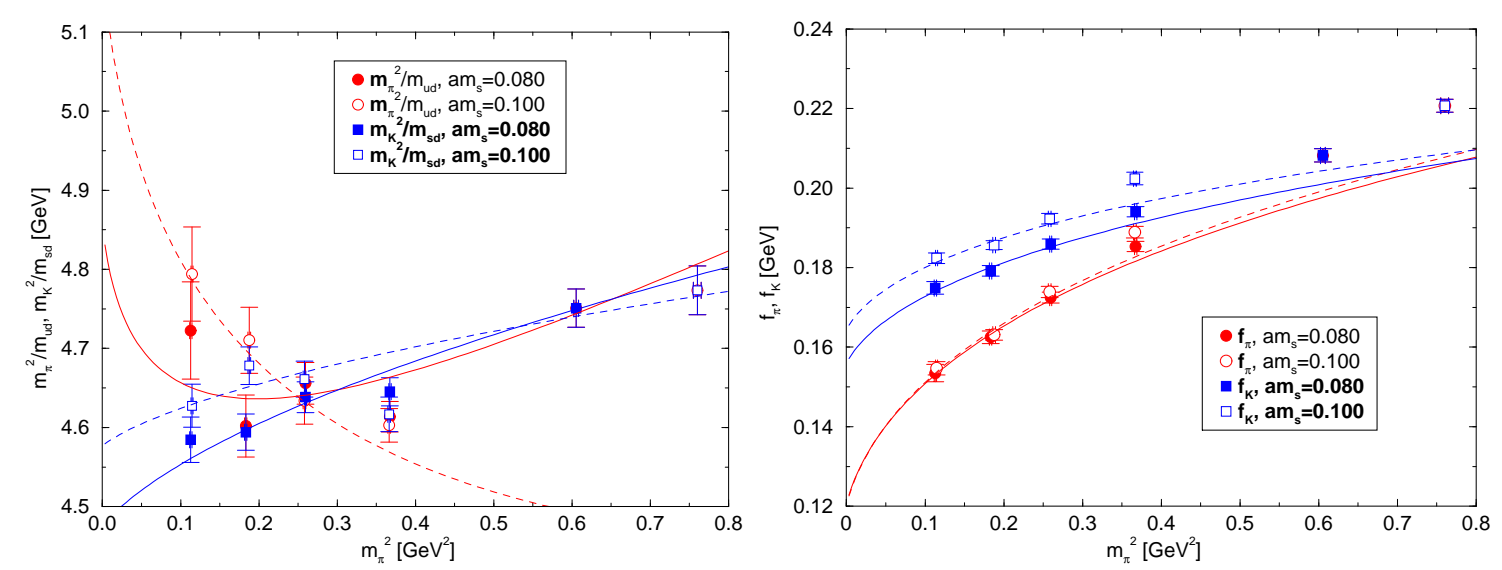

Figure 5: Chiral extrapolation using the NLO reduced $S U(2)$ ChPT formulae. The organization is same as Figure 4.

whose expressions are too involved to present here [ए6]. Among relevant SU(3) LECs, $L_{1}^{r}, L_{2}^{r}, L_{3}^{r}$ and $L_{7}^{r}$ which appear only in the NNLO contributions cannot be determined precisely. We introduce values $L_{1}^{r}=(0.43 \pm 0.12) \cdot 10^{-3}, L_{2}^{r}=(0.73 \pm 0.12) \cdot 10^{-3}, L_{3}^{r}=(-2.53 \pm 0.37) \cdot 10^{-3}$ and $L_{7}^{r}=(-0.31 \pm 0.14) \cdot 10^{-3}$ (defined at $\mu=770 \mathrm{MeV}$ ) from a phenomenological estimate [ए]] and determine others $L_{4}^{r}, L_{5}^{r}, L_{6}^{r}$ and $L_{8}^{r}$ by a fit. Thus, the chiral extrapolation with (4.Cl)-(4.4) contains 16 fit parameters in total.

We fit $m_{\pi}^{2} / m_{u d}, m_{K}^{2} / m_{s d}, f_{\pi}$ and $f_{K}$ simultaneously taking the correlation within the same sea quark mass $\left(m_{u d}, m_{s}\right)$ into account. By using all data points, reasonable quality of the fit is obtained with $\chi^{2} /$ dof $=2.52$. In this new study, we determine the lattice scale by the result of $f_{\pi}$ extrapolated to the physical point with the input $f_{\pi}=130.0 \mathrm{MeV}$. As a result, we obtain $a^{-1}=1.968$ (39) GeV and the pion mass covers the range of $340 \mathrm{MeV}<m_{\pi}<870 \mathrm{MeV}$. Figure G shows all quantities in question as a function of $m_{\pi}^{2}$. Different symbols correspond to the pion data $\left(m_{\pi}^{2} / m_{u d}\right.$ and $\left.f_{\pi}\right)$ and the kaon data $\left(m_{K} / m_{s d}\right.$ and $\left.f_{K}\right)$ while the filled (open) symbols represent a fixed lighter (heavier) strange quark mass, which is accompanied by the solid (dashed) curves.

Extrapolating the data to the physical point $\left(\xi_{\pi}^{\text {(phys) }}, \xi_{K}^{(\text {phys })}\right)$, which is determined with $m_{\pi}=$ 135.0 MeV, $m_{K}=495.0 \mathrm{MeV}$ and $f_{\pi}=130.0 \mathrm{MeV}$, we obtain preliminary results $m_{u d}^{\overline{\mathrm{MS}}}(2 \mathrm{GeV})=$ 3.64(12) MeV, $m_{s}^{\overline{\mathrm{MS}}}(2 \mathrm{GeV})=104.5(1.8) \mathrm{MeV}, m_{s} / m_{u d}=28.71(52), f_{K}=157.3(5.5) \mathrm{MeV}$ and $f_{K} / f_{\pi}=1.210(12)$, where the errors are statistical only.

In order to discuss the convergence property of ChPT as in the case of $N_{f}=2$, we need to determine individual LECs with a high accuracy. However, with the data points obtained for two different strange quark masses, we have a limited constraint about the $\xi_{K}$ dependence hence large errors for LECs. From the phenomenological side, it is advantageous to determine LECs along the line of this work because the results can be used as inputs in the calculation of different quantities including $B_{K}$ and $K l_{3}$ form factors. For these motivation, we are planning to extend the chiral extrapolation with more data points with $m_{u d}=m_{s}$. 

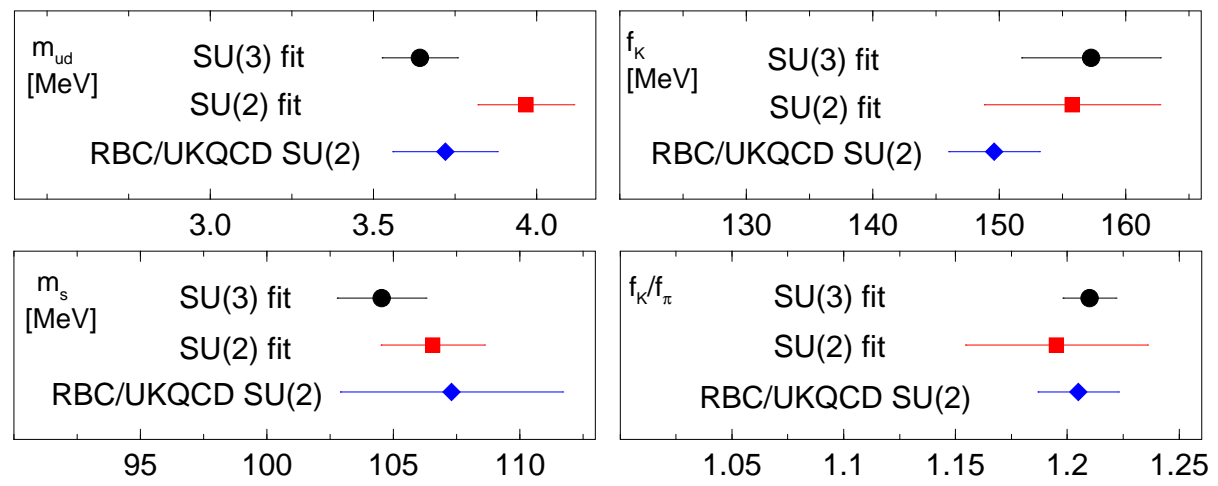

Figure 6: Comparison of physical values between results from NNLO $S U(3)$ analysis, NLO reduced $S U(2)$ analysis and results obtained by UKQCD and RBC Collaborations [प⿴囗\zh25).

\subsection{Fit to the reduced $S U(2)$ ChPT to NLO}

As a check of the chiral extrapolation we carried out with the NNLO ChPT, we also study different fit ansatz. It is also possible to carry out the extrapolation to the physical point $\xi_{\pi}^{\text {(phys) }}$ by paying attention only to the dependence of the data on the up-down quark mass, or the pion mass. Integrating out the strange quark as a static heavy quark, one obtain an effective theory which

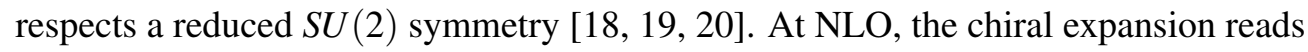

$$
\begin{aligned}
m_{\pi}^{2} / m_{u d} & =2 B\left(1+\frac{1}{2} \xi_{\pi} \ln \xi_{\pi}\right)+c_{3} \xi_{\pi}, \\
m_{K}^{2} / m_{s d} & =2 B^{(K)}+c_{1}^{(K)} \xi_{\pi} \\
f_{\pi} & =f\left(1-\xi_{\pi} \ln \xi_{\pi}\right)+c_{4} \xi_{\pi} \\
f_{K} & =f^{(K)}\left(1-\frac{3}{8} \xi_{\pi} \ln \xi_{\pi}\right)+c_{2}^{(K)} \xi_{\pi},
\end{aligned}
$$

where we have LECs $B^{(K)}, f^{(K)}, c_{1}^{(K)}$ and $c_{2}^{(K)}$ in addition to the $S U(2)$ LECs appeared in Section B]. In the present case, all LECs depend on strange quark mass. With the lightest three $m_{u d}$ points, which are in the valid region of this framework, i.e. $m_{u d} \ll m_{s}$ for each fixed value of $m_{s}$, we carry out the correlated fit for the quantities sharing the same mass point $\left(m_{u d}, m_{s}\right)$. Figure $\square$ shows the fit curves obtained in this way.

The fit results for each fixed $m_{s}$ are extrapolated to the physical strange quark mass $m_{s}^{\text {(phys), }}$ which is determined by solving $m_{K}^{2} /\left.m_{s}\right|_{\xi_{\pi} \text { (phys) }}=(495.0 \mathrm{MeV})^{2} / m_{s}$. In Figure $\mathbf{6}$, we compare physical results for $m_{u d}, m_{s}, f_{K}$ and $f_{K} / f_{\pi}$ from the full NNLO $S U$ (3) ChPT (circles), and from the NLO reduced $S U(2)$ ChPT (squares from our analysis and diamonds from the similar analysis by RBC and UKQCD Collaborations [ㅁ]]). The agreement among different fitting prescription is encouraging.

\section{Summary}

We tested the convergence property of ChPT by comparing the analytic prediction with the lattice data obtained in the dynamical simulation with the overlap fermions. For $N_{f}=2$, we carried 
out the chiral fit to the NLO and NNLO formulae and compare three different expansion parameters. We found that ChPT at NLO does not converge around the scale of kaon mass. It implies that one must take the NNLO effects into account to deal with the pion and kaon data points in an equal footing. In the $N_{f}=2+1$ simulation, we fitted the data to the ChPT prediction to NNLO for the first time. The validity of the extrapolation to the physical mass point is checked with the results from the fit with the reduced $S U(2)$ ChPT. We are planning to increase the data point with $m_{u d}=m_{s}$ to obtain the $S U(3)$ LECs with high accuracy for a detailed study of the convergence property.

Numerical simulations are performed on Hitachi SR11000 and IBM System Blue Gene Solution at High Energy Accelerator Research Organization (KEK) under a support of its Large Scale Simulation Program (Nos. 07-16 and 08-05 ). This work is supported in part by the Grant-in-Aid of the Ministry of Education (No. 20105005).

\section{References}

[1] G. Colangelo, in these proceedings.

[2] H. Neuberger, Phys. Lett. B427 (1998) 353

[3] JLQCD Collaboration (S. Aoki et al.), Phys. Rev. D 78 (2008) 014508,

[4] JLQCD and TWQCD Collaborations (H. Matsufuru et al), PoS LAT2008 (2008) 077.

[5] JLQCD Collaboration (S. Aoki et al.), Phys. Rev. D 77 (2008) 094503.

[6] JLQCD and TWQCD Collaborations (J. Noaki et al.), arXiv:0806.0894 [hep-lat].

[7] T. DeGrand and S. Schaefer, Comp. Phys. Comm. 159 (2004) 185.

[8] L. Giusti, P. Hernández, M. Laine, P. Weisz, H. Wittig, JHEP 04 (2004) 013.

[9] S. Aoki, H. Fukaya, S. Hashimoto and T. Onogi, Phys. Rev. D 76 (2007) 054508.

[10] G. Colangelo, S. Dürr and C. Haefeli, Nucl. Phys. B721 (2005) 136.

[11] R. Brower, S. Chandrasekharan, J. W. Negele and U.-J. Wiese, Phys. Lett. B560 (2003) 64.

[12] JLQCD and TWQCD Collaborations (S. Aoki et al.), Phys. Lett. B665 (2008) 294; JLQCD and TWQCD Collaborations (T.W. Chiu et al.), PoS (LATTICE 2008) (158) .

[13] G. Martinelli, C. Pittori, C. T. Sachrajda, M. Testa and A. Vladikas, Nucl. Phys. B445 (1995) 81.

[14] JLQCD and TWQCD Collaborations (J. Noaki et al.), arXiv:0907.2751 [hep-lat].

[15] G. Colangelo, J. Gasser and H. Leutwyler, Nucl. Phys. B603 (2001) 125.

[16] G. Amorós, J. Bijnens and P. Talavera, Nucl. Phys. B568 (2000) 319. We thank J. Bijnens for providing his Fortran code evaluating the contribution of sunset integrals among the two-loop effects.

[17] G. Amorós, J. Bijnens and P. Talavera, Nucl. Phys. B602 (2001) 87, [arXiv:hep-ph/0101127].

[18] J. Gasser, C. Haefeli, M. A. Ivanov and M. Schmid, Phys. Lett. B652 (2007) 21.

[19] UKQCD and RBC Collaborations (C. Allton et al.), Phys. Rev. D 78 (2008) 114509.

[20] PACS-CS Collaborations (S. Aoki et al.), [arXiv:0807.1661 [hep-lat]]. 